\title{
Heart failure in sinus rhythm as the sole manifestation of Graves' disease
}

\author{
B.P. O’Malley, B.J. Northover ${ }^{1}$ and K.L. Woods
}

Department of Pharmacology and Therapeutics, University of Leicester and ${ }^{1}$ Department of Pharmacy, Leicester Polytechnic, Leicester, UK.

\begin{abstract}
Summary: A middle-aged man with Graves' disease presented with heart failure in sinus rhythm which preceded the more typical manifestations of the disease by some months. Once suspected, the diagnosis was supported by measurement of systolic time intervals, enabling the instigation of treatment in advance of biochemical confirmation.
\end{abstract}

\section{Introduction}

It is well recognized that the heart is highly sensitive to circulating thyroid hormones and that a rapid pulse is virtually a sine qua non of thyrotoxicosis. Except in the elderly, however, the cardiovascular manifestations of thyrotoxicosis are usually less notable than the metabolic and ocular changes. In young patients the myocardium usually has no difficulty in meeting the extra circulatory demands, overt cardiac failure only occurring if there is a complicating factor such as uncontrolled atrial fibrillation or ischaemic or rheumatic heart disease. In older patients the clinical diagnosis of thyrotoxicosis and attendant heart failure may be missed because the thyroid gland may not be enlarged and eye signs are usually absent (Lahey, 1931).

Systolic time intervals, a measure of left ventricular contractility, provide an index of the peripheral actions of thyroid hormones (Paulus et al., 1980; Parisi et al., 1974). We describe a middle-aged man with Graves' disease whose presentation with heart failure in sinus rhythm preceded the more typical manifestations of the disease by some months. Once suspected, the diagnosis was supported by measurement of the systolic time intervals enabling the instigation of treatment in advance of biochemical confirmation.

\section{Case report}

A 50 year old man was referred with a 2 year history of dyspnoea on exertion, increasing in the 3 months

Correspondence: B.P. O'Malley, M.R.C.P., Department of Pharmacology, Clinical Sciences Building, Leicester Royal Infirmary, Leicester LE2 7LX, UK.

Accepted: 16 June 1986 before presentation and associated in that time with orthopnoea and bilateral ankle swelling. There had been no other symptoms, in particular no chest pain or weight loss. There was no family history of thyroid disease. He was a non-smoker and did not drink alcohol.

On examination the heart rate was $96 /$ minute in sinus rhythm and the blood pressure was $115 /$ $70 \mathrm{mmHg}$. The jugular venous pressure was $1 \mathrm{~cm}$ raised and there was bilateral pitting oedema of the ankles. The apex beat was not displaced but forceful and there was a gallop rhythm. There were no murmurs audible and his chest was clear. There was no goitre, lid lag or exophthalmos.

A provisional diagnosis of congestive cardiac failure was made, presumed due to ischaemic heart disease. Initial investigations revealed a normal full blood count but a raised plasma viscosity of $1.85 \mathrm{cP}$. A biochemical screen including cardiac enzymes and a fasting lipid profile was normal apart from a raised adjusted calcium of $2.81 \mathrm{mmol} / 1$ (normal range 2.1-2.6) with a normal phosphate and alkaline phosphatase and a raised random glucose of $7.7 \mathrm{mmol} / 1$. The electrocardiogram displayed a sinus tachycardia of $100 / \mathrm{min}$ but was otherwise normal. On chest X-ray, heart size was at the upper limit of normal (cardiothoracic ratio $50 \%$ ) and the lung fields appeared clear.

When reviewed 6 weeks later on frusemide, he had lost $6.6 \mathrm{~kg}$ in weight and complained of increasing fatigue. Examination then revealed a heart rate of 120 / minute, sinus rhythm. The apex beat was again forceful but not displaced and the jugular venous pressure was not elevated. There was no longer a third heart sound and the ankle swelling had settled. A further chest X-ray and electrocardiogram were both normal. However, marked deterioration occurred 


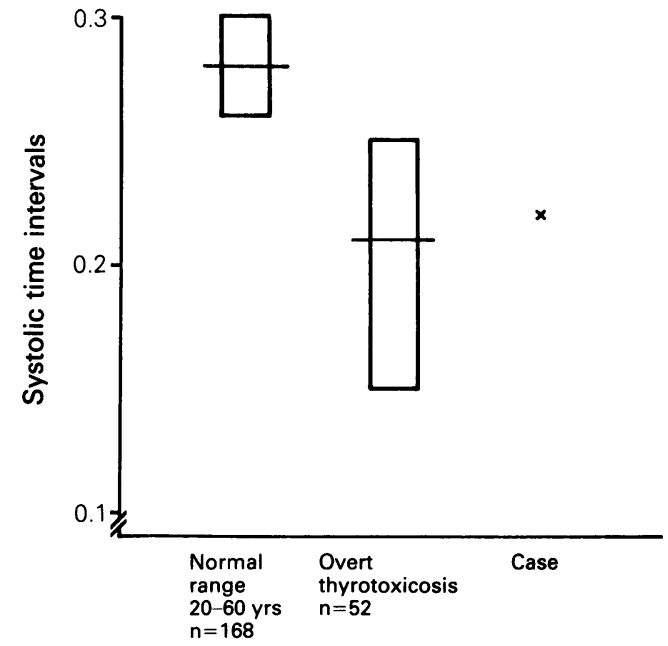

Figure 1 Systolic time intervals (ratio of pre-ejection period/left ventricular ejection time) in 168 euthyroid individuals and 52 patients with thyrotoxicosis. $x=$ the value obtained in the reported case.

over the following month with weight loss of $7 \mathrm{~kg}$ and the development of heat intolerance, bouts of sweating, intermittent diarrhoea and proximal muscle weakness. He was admitted to hospital with the presumptive diagnosis of thyrotoxicosis. Whilst awaiting the results of thyroid hormone assays (serum thyroxine, triiodothyronine and thyroid stimulating hormone), systolic time intervals were measured (see Figure 1). The pre-ejection period/left ventricular ejection time ratio of 0.23 indicative of a high output state corroborated the clinical diagnosis. On this evidence, propranolol $40 \mathrm{mg}$ orally t.d.s. and carbimazole $15 \mathrm{mg}$ orally t.d.s. were commenced. A pretreatment technetium thyroid scan had revealed uniform uptake in a normal sized thyroid gland. Over 24 hours, he improved symptomatically and, since there was no evidence of recurrent heart failure, propranolol was increased to $80 \mathrm{mg}$ orally t.d.s., resulting in a heart rate of 80 per minute. Initial serum thyroxine and triiodothyronine levels were $275 \mathrm{nmol} / 1$ (normal range $54-142$ ) and $8.5 \mathrm{nmol} / 1$ (normal range $0.8-2.5$ ) respectively and the thyroid stimulating hormone (Delfia) was suppressed at less than $0.05 \mathrm{mU} / 1$ (normal range $0.3-3.8$ ) confirming thyrotoxicosis. Thyroglobulin $(1: 2560)$ and thyroid microsomal
$(1: 25600)$ antibodies were detected and thyroid stimulating antibodies (TRAK kit) registered $30 \%$, in keeping with Graves' autoimmune thyroiditis. His subsequent recovery was uneventful.

\section{Discussion}

The detection of masked hyperthyroidism, otherwise termed apathetic, cryptic or occult, undoubtedly requires a high index of suspicion. The majority of such cases, many of whom are elderly women, present with cardiac enlargement or failure but have neither goitre nor eye signs (Lahey, 1931). Graves' thyroiditis is very seldom so enigmatic, occurring mainly in younger individuals and displaying the classic features of exophthalmos, goitre, heat intolerance, tremor and bounding pulse. Thyrotoxic heart failure is usually confined to elderly patients and then generally in association with atrial fibrillation or other predisposing factors, for instance valvular disease or myocardial ischaemia. The case of Graves' thyroiditis we describe is unusual on several counts. Firstly, heart failure developed in sinus rhythm and without other demonstrable cardiac disease. Secondly, the failure preceded the more typical systemic manifestations of weight loss, diarrhoea and heat intolerance by many months.

It can be difficult, as here, to distinguish clinically the vigorous apex beat of a high output state from the dyskinetic apex of an impaired left ventricle. Systolic time intervals provide a non-invasive measure of left ventricular contractility, well established as a reliable guide to tissue thyroid status (Paulus et al., 1980; Parisi et al., 1974). The availability of the technique made it possible to confirm that contractility was increased within hours of admission and thus allowed treatment to be started before thyroid hormone levels were available. Beta blockade is now well recognized as providing rapid symptomatic relief in thyrotoxicosis (Riddle \& Schwartz, 1972) and might reasonably be expected to be beneficial in thyrotoxic heart failure. It was certainly effective in the patient we report. However, our previous observation of propranololinduced failure in two thyrotoxic patients with no previous history of myocardial ischaemia (O'Malley et al., 1982) suggests that its introduction should be gradual and closely monitored.

We suggest that in any patient presenting with heart failure of indefinite aetiology serum thyroid hormone measurements are mandatory.

\section{References}

LAHEY, F.H. (1931). Non-activated (apathetic) type of hyperthyroidism. New England Journal of Medicine, 204, 747.

O'MALLEY, B.P., ABBOTT, R.J., BARNETT, D.B., NORTHOVER, B.J. \& ROSENTHAL, F.D. (1982). Propranolol versus carbimazole as the sole treatment for thyrotoxicosis. A 
consideration of circulating thyroid hormone levels and tissue thyroid function. Clinical Endocrinology (Oxford), $16,545$.

PARISI, A.F., HAMILTON, B.P. \& THOMAS, C.N. (1974). The short cardiac pre-ejection period: an index to thyrotoxicosis. Circulation, 49, 900.
PAULUS, J.W., RANQUIN, R. \& PARIZEL, G. (1980). Systolic time intervals: a valuable parameter of thyroid function. Angiology, 31, 100.

RIDDLE, M.C. \& SCHWARTZ, T.B. (1972). New tactics for hyperthyroidism: sympathetic blockade. Annals of Internal Medicine, 72, 749. 